The Astrophysical Journal, 323:L73-L76, 1987 December 1

1987. The American Astronomical Society. All rights reserved. Printed in U.S.A.

\title{
DIRECT IMAGING AT 12 MICRONS OF THE STAR-FORMING REGION W51 IRS 2
}

\author{
John Bally, ${ }^{1}$ John F. Arens, ${ }^{2}$ Roger Ball,,${ }^{2,3}$ Robert Becker, ${ }^{3,4}$ AND John LaCy ${ }^{5}$ \\ Received 1987 July 13; accepted 1987 August 28
}

\begin{abstract}
We have made direct imaging observations of the luminous star-forming region W51 IRS 2 with the 10 by 64 pixel Berkeley direct readout infrared camera. Using the Steward Observatory 90 inch $(2.3 \mathrm{~m})$ reflector at 12 $\mu \mathrm{m}$, we mosaicked four adjacent fields to obtain a 2500 pixel map with a pixel size of $00^{\prime \prime} .4$ and a resolution better than 1". The distribution of the $12 \mu \mathrm{m}$ emission shows a symmetric "dumbbell" in which the central and brightest structure is $\sim 4^{\prime \prime} \times 1^{\prime \prime}$ in size. The observed surface brightness is consistent with optically thick 12 $\mu \mathrm{m}$ emission from dust at radiative equilibrium with a $3 \times 10^{6} L_{\odot}$ central source. These results suggest that W51 IRS 2 is a hot $\left(T \approx 150 \mathrm{~K}\right.$ ), very dense $\left(n \geq 10^{7} \mathrm{~cm}^{-3}\right)$ massive disk (of order 100-200 $\left.M_{\odot}\right)$ surrounding a very luminous and massive protostellar source.
\end{abstract}

Subject headings: infrared: sources - stars: formation

\section{INTRODUCTION}

The intense radio source W51 is among the Galaxy's most luminous star-forming regions and one of the brightest in the sky at mid-infrared and radio wavelengths. Two intense centers of activity have been identified in this region: W51 IRS 1 and W51 IRS 2, which has also been called W51 North. We discuss only W51 IRS 2 in this Letter. An intense $\mathrm{H}_{2} \mathrm{O}$ maser in the region was detected by Genzel and Downes (1977) and subsequently reobserved with VLBI (Downes et al. 1979; Genzel et al. 1981, Schneps et al. 1981). The proper motions of individual maser knots permitted a direct measurement of the distance to this source, $7.5 \mathrm{kpc}$, which agrees well with the kinematic distance (Bieging 1975). The cluster of maser emitting knots revealed the presence of a very complex, high-velocity outflow with an expansion velocity of at least $50 \mathrm{~km} \mathrm{~s}^{-1}$. The maser emission shows hierarchical clustering and a hint of bipolarity in two nearly orthogonal directions. In a $6 \mathrm{~cm}$ radio continuum map of this region, Genzel et al. (1982) found a compact H II region displaced by $\sim 3^{\prime \prime}$ northwest from the center of the water maser activity.

The infrared luminosity of W51 IRS 2 has been estimated to be $3 \times 10^{6} L_{\odot}$ in a $30^{\prime \prime}$ beam (Thronson and Harper 1979; Erickson and Tokunaga 1980). Several groups have produced mid-infrared maps of this region with single pixel detectors. Genzel et al. (1982) presented both 8 and $20 \mu \mathrm{m}$ maps of the W51 IRS 2 region with a resolution of $\sim 2$ "' 5 . These images revealed two components separated by 4 " from each other, which they called IRS 2 East and IRS 2 West. The latter was found to coincide with a slightly extended $\left(5^{\prime \prime}\right.$ or $\left.0.2 \mathrm{pc}\right) 2 \mathrm{~cm}$ continuum source which is presumed to be a compact $\mathrm{H}$ II region. In the Genzel et al. maps, IRS 2 West appears much fainter than IRS 2 East at $8 \mu \mathrm{m}$, and perhaps $30 \%$ fainter at

\footnotetext{
${ }^{1}$ AT \& T Bell Laboratories, Holmdel, NJ.

${ }^{2}$ Space Sciences Laboratory, University of California, Berkeley.

${ }^{3}$ Institute of Geophysics and Planetary Physics, Lawrence Livermore National Laboratory, Livermore, CA.

${ }^{4}$ University of California, Davis.

${ }^{5}$ University of Texas, Austin.
}

$20 \mu \mathrm{m}$. The two components are also distinguished by the observation of deep silicate absorption toward the eastern, but not the western, peak. The larger scale 10 and $20 \mu \mathrm{m}$ maps produced by Hackwell, Grasdalen, and Gehrz (1982) also show an elongated source with a major axis oriented at a position angle of $\sim 120^{\circ}$. At near-infrared wavelengths, Beckwith and Zuckerman (1982) detected emission in the 2 $\mu \mathrm{m} \mathrm{H}_{2} S(1)$ line of molecular hydrogen, but with a total luminosity only $4 \%$ of that of the shocked $\mathrm{H}_{2}$ emission in the $\mathrm{BN} / \mathrm{KL}$ region located behind the Orion Nebula.

In this Letter we present high-resolution images of this region at a wavelength of $12 \mu \mathrm{m}$, obtained with the Berkeley direct readout (DRO) array camera.

\section{INSTRUMENTATION AND OBSERVATIONS}

The camera used in acquiring these data is a slight modification of one that is discussed at some length elsewhere (Arens et al. 1987). Because the use of this kind of instrument for astronomical observations is still rather novel, we will describe it briefly here.

The camera optics and detector array are housed in a side-looking dewar which uses liquid helium to cool the detector chip to its normal operating temperature of 8-12 K. The optical layout in the dewar employs an entrance window and two converging lenses, all of $\mathrm{ZnSe}$, a cold stop, and filters. The first lens, of focal length $f_{1}$, is located $f_{1}$ downstream of the telescope focus and $f_{1}$ upstream of the cold stop. The secondary mirror of the telescope is thus imaged onto the cold stop, whose diameter is matched to this image for stray light rejection. Because light passes through the filters and, subsequently, the cold stop as a parallel beam, internal reflections in the filters are of no consequence. The filters have been placed upstream of the cold stop to suppress a ghost image caused by reflection from the detector surface to the filters and back.

The detector array is a hybrid device manufactured by the Hughes Aircraft Company, composed of an infrared-sensitive, pixelated gallium-doped silicon die and a readout chip with an amplifier for each pixel. Each pixel is $120 \mu \mathrm{m}$ square, and 
the array consists of 640 pixels laid out in a $10 \times 64$ format. Preliminary laboratory tests of the detector chip have indicated the quantum efficiency to be $\sim 15 \%$. The amplifiers on the readout chip are connected to the detector pixels via indium bump bonds. A correlated double sampling technique (Arens et al. 1987) is used to measure the integrated signal from each pixel with low readout noise. The circuit is designed for fast readout cycles (of order $1 \mathrm{~ms}$ ) in order to avoid saturation of the pixels by the large thermal foreground flux. The data volume is decreased in real time by the use of a digital co-adder which, like the timing generator for the chip, is under software control for maximum flexibility.

The observations reported here were made on the night of 1986 June 20 with the camera mounted on the 90 inch telescope of the Steward Observatory. The optics were configured to provide a scale of 0.39 per pixel at the detector, and the camera was operated with the long axis of the detector array aligned north and south. A circular variable filter was used to give a system with $10 \%$ fractional bandwidth centered at $12.0 \mu \mathrm{m}$. Data were taken in an alternating cycle of $8 \mathrm{~s}$ on-source followed by $8 \mathrm{~s}$ with the telescope pointed at a blank position $1^{\prime}$ north. Four such cycles were used to make each $10 \times 64$ panel of the mosaicked image of W51 IRS 2, which contains four of these panels. The final image thus has $32 \mathrm{~s}$ of on-source integration time at every point, and a total of $256 \mathrm{~s}$ of observing time were used to make the map. The off-source data were used to estimate the foreground contribution, which was subtracted from the on-source observations. Variations in the foreground flux on a time scale faster than the on-off source cycling were tracked by measurements of pixels in parts of the frame away from detectable W51 flux, and the data were corrected for this effect. The final image has a resolution of just under $1^{\prime \prime}$ (FWHM).

The instrumental gain was calibrated by observations of the bright stars $\beta$ Peg and $\delta$ Oph before and after the program source. The $12 \mu \mathrm{m}$ flux of the former was taken to be $292 \mathrm{Jy}$, on a system where the flux of Vega at this wavelength is 27.9 Jy (Hackwell and Gehrz 1974; Leggett 1985). This was used as the photometric calibration. On this scale we measure a flux of $210 \mathrm{Jy}$ from our observations of $\delta$ Oph, compared to $170 \mathrm{Jy}$ measured at $11.4 \mu \mathrm{m}$ for the AFCRL survey (Walker and Price 1975) with the same normalization. The absolute flux calibration of our data is thus uncertain at the level of $25 \%-30 \%$. However, the relative brightnesses of different points in the map are much better determined. The blank sky data were used to flat-field the final image. Although offsets between adjacent panels of the final image are smaller than the noise level, a small-amplitude fixed pattern of north-south stripes, corresponding to every other column of detector elements, remains in the data.

\section{RESULTS}

Figure 1 shows a contour map of the $12 \mu \mathrm{m}$ emission from the infrared source W51 IRS 2. The observed intensity is distributed in a symmetric, dumbbell-shaped structure. The aspect ratio (major axis to minor axis ratio) of this object is $\sim 4$ to 1 in its inner and brightest parts, which contain two nearly equal peaks connected by a well-resolved, elongated envelope that forms the bar of the dumbbell. The position

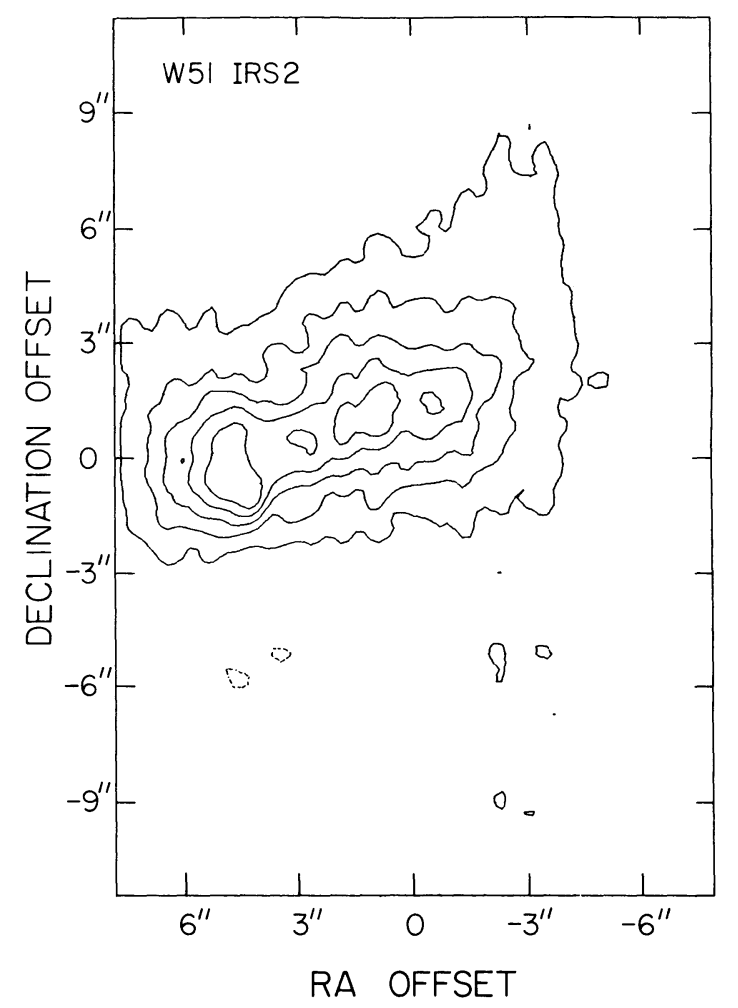

FIG. 1.-A contour map showing the intensity of $12 \mu \mathrm{m}$ radiation in W51 IRS 2. Contours are plotted at intervals of $2 \mathrm{Jy}$ per square arcsecond. Positional offsets refer to the map center, the absolute position of which is not well determined. The rms noise in this map is $\sim 0.8 \mathrm{Jy}$ per square arcsecond.

angle of its major axis is $\sim 110^{\circ}$, similar to the structure seen by Hackwell, Grasdalen, and Gehrz (1982). However, our results differ in detail from the infrared maps of Genzel et al. (1982) in that the intensity distribution in our map shows a high degree of symmetry about the geometric center of the source. The maximum surface brightness is $11.9 \mathrm{Jy}$ per square arcsecond in the eastern peak, $11.8 \mathrm{Jy}$ per square arcsecond in the western. The rms noise in blank areas of the map is $\sim 0.8$ Jy per square arcsecond. The two peaks are separated by $\sim 4^{\prime \prime}$, or $30,000 \mathrm{AU}$ at the assumed distance of $7.5 \mathrm{kpc}$. The total flux of W51 IRS 2 in our map is $490 \mathrm{Jy}$. Figure 2 (Plate L2) shows a gray scale representation of the data.

The observed $12 \mu \mathrm{m}$ size and flux of W51 IRS 2 are consistent with the expected source properties if the midinfrared radiation is emitted by dust in radiative equilibrium with a radiation field that corresponds to the luminosity of the W51 IRS 2 region, $L=3 \times 10^{6} L_{\odot}$ (Erickson and Tokunaga 1980). This can be shown using the results of Scoville and Kwan (1976), who studied the transfer of radiation through a spherical dust cloud at temperature $T_{d}$. From their equation (9), the radial dependence of $T_{d}$ is

$$
\begin{aligned}
T_{d}(r)= & 210 \mathrm{~K}\left(\frac{r}{2 \times 10^{17} \mathrm{~cm}}\right)^{-2 / 5} \\
& \times\left(\frac{L}{3 \times 10^{6} L_{\odot}}\right)^{1 / 5}\left(\frac{f}{0.02}\right)^{-1 / 5}
\end{aligned}
$$




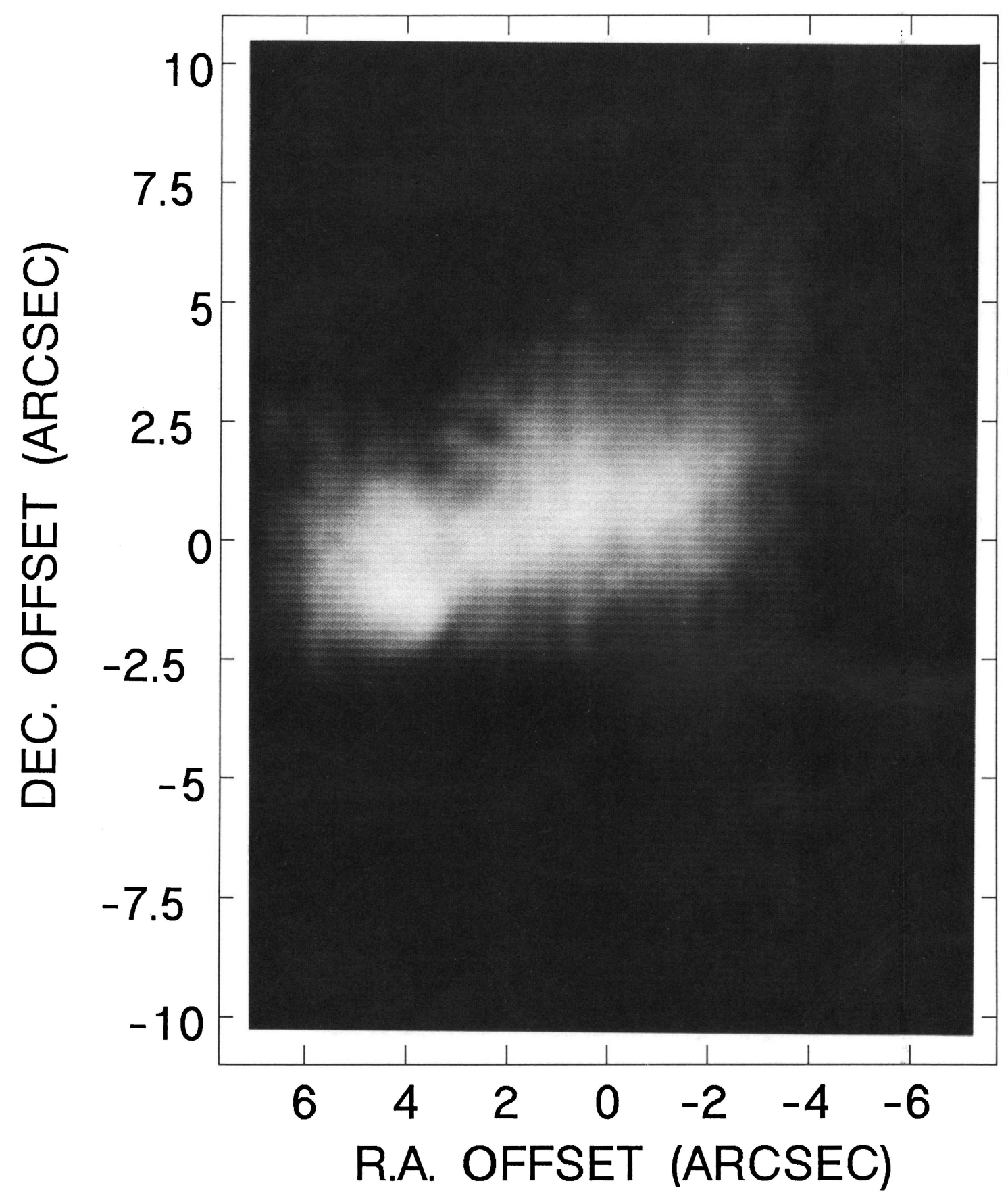

FIG. 2.-A photographic gray scale representation of the same data as shown in Fig. 1 Bally, Arens, Ball, Becker, and Lacy (see 323, L74) 
for a dust emissivity proportional to $\lambda^{-1}$. The parameter $f$ is the dust emissivity at $50 \mu \mathrm{m}$ (some authors use the term "absorption efficiency" for this parameter). The three quantities in the denominator of this expression are best estimates for W51, with $f \approx 0.02$, which is somewhat lower than the value, $f=0.09$, used by Scoville and Kwan to model the Orion core. The radius where most of the radiation is absorbed, $r$, is assumed to correspond to one half of the $4^{\prime \prime}$ separation between the peaks in our map. If the emissivity dependence were $\lambda^{-2}$, the functional form of the above expression would be slightly changed and $T_{d}$ would be decreased for the W51 IRS 2 source, to $140 \mathrm{~K}$. Over a reasonable range of dust parameters, $T_{d}$ lies in the range $140-210 \mathrm{~K}$. These results assume that the dust is optically thin at $100 \mu \mathrm{m}$. If the optical depth at $100 \mu \mathrm{m}$ is actually $\sim 0.5$ as suggested by Thronson and Harper (1979) and by the maps of Harvey et al. (1986), the results shown in Figure 2 of Scoville and Kwan suggest that the temperature at $r=2 \times 10^{17} \mathrm{~cm}$ may be lower by $\sim 30 \%$, giving a temperature at our chosen radius between 100 and $150 \mathrm{~K}$. These results are largely independent of the detailed geometry of the dust cloud.

The presence of material at high temperatures has previously been inferred from observations of high-excitation $\mathrm{NH}_{3}$ transitions (Ho, Genzel, and Das 1983; Mauersberger et al. 1986, 1987) and from the 10-20 $\mu \mathrm{m}$ colors (Hackwell, Grasdalen, and Gehrz 1982). Such hot dust radiates effectively at $12 \mu \mathrm{m}$ since the Planck function peaks near $20 \mu \mathrm{m}$ in this temperature range. Mauersberger et al. find that the ammonia emission requires that there be some material at very high temperatures and densities $\left(T \geq 250 \mathrm{~K}, n \geq 10^{7} \mathrm{~cm}^{-3}\right)$, implying a molecular hydrogen column density $N\left(\mathrm{H}_{2}\right)=2 \times$ $10^{24} \mathrm{~cm}^{-2}$ for a characteristic source size of $2 \times 10^{17} \mathrm{~cm}$. Alternatively, Jaffe, Becklin, and Hildebrand (1984) estimate a column density of $1 \times 10^{24} \mathrm{~cm}^{-2}$ directly from their 400 $\mu \mathrm{m}$ observations, using the conversion from submillimeter optical depth to column density discussed by Hildebrand (1983).

At these column densities, the optical depth at $12 \mu \mathrm{m}$ is certainly much greater than one; Jaffe, Becklin, and Hildebrand estimate that the extinction at $20 \mu \mathrm{m}$ is $1.5-6$ mag. Therefore, our image approximately probes the surface of unit opacity, where emission properties are like those of a blackbody. We can use our observed surface brightness directly to estimate the dust temperature at the $\tau=1$ surface. The peak surface brightness of $11.9 \mathrm{Jy}$ per square arcsecond would give $T=112 \mathrm{~K}$, fairly close to our earlier estimate from the simple models of Scoville and Kwan (1976) using the observed source size and luminosity. The energy requirements of the source therefore are generally consistent with a model in which the luminosity of one or more central objects is reradiated in the thermal infrared by optically thick dust distributed in a disk or torus of mean radius $2 \times 10^{17} \mathrm{~cm}$.

We consider two possible models for the observed intensity distribution:

1. The $12 \mu \mathrm{m}$ source consists of two distinct cloud cores, each heated by a different group of luminous young stellar objects. Each $12 \mu \mathrm{m}$ peak contains its own source of luminosity. The apparent symmetry of the source then has no particular significance. This picture is similar to that proposed by Genzel et al. (1982).

2. The source is a nearly edge-on disk surrounding a single group of luminous young stellar objects. This group lies in between the two $12 \mu \mathrm{m}$ peaks.

In favor of the first possibility, we note that the size scale of the bright $12 \mu \mathrm{m}$ emission is comparable to the size of the infrared $\mathrm{BN} / \mathrm{KL}$ nebula in Orion, although the luminosity of W51 is more than one order of magnitude greater. Our experience with luminous star-forming complexes in the solar neighborhood is that most such regions contain not one but a cluster of relatively luminous protostellar or young stellar objects. However, the infrared emission from such clusters is generally very lumpy and does not show a high degree of symmetry. The regularity of the $12 \mu \mathrm{m}$ distribution observed in W51 IRS 2 must be a matter of chance in this model.

In the interpretation of Genzel et al. (1982), IRS 2 East is a very dense, active star-forming cloud core, exhibiting deep silicate absorption, while the western peak is a lower column density and more evolved region whose mid-infrared emission is produced by dust embedded in the observed compact $\mathrm{H}$ II region (Genzel et al. 1981). This morphology is similar to the situation in Orion, with $\mathrm{BN} / \mathrm{KL}$ being the dense, active core and the Trapezium being the second and older center of activity. We find that the western source in W51 has a similar surface brightness to the eastern source. As demonstrated above, the observed fluxes imply high opacity in the midinfrared if the dust is in radiative equilibrium. It is possible that the dust in the western source is much hotter and has lower opacity. However, our results are fully consistent with the idea that the observed $12 \mu \mathrm{m}$ symmetry is related to a real, physical symmetry of the source.

This leads us to explore the second possibility, that the highly symmetric $12 \mu \mathrm{m}$ structure is a nearly edge-on disk of hot gas and dust whose center contains a highly obscured source or small cluster of sources which produce most of the luminosity in this region and which are probably responsible for the maser activity. The most attractive feature of this model is that it provides a natural explanation for the observed morphology. The mass of the disk may be estimated from the radius $\left(2 \times 10^{17} \mathrm{~cm}\right)$ and thickness $\left(1 \times 10^{17} \mathrm{~cm}\right)$, with the density taken to be $10^{7} \mathrm{~cm}^{-3}$ from the ammonia observations. This calculation gives a disk mass of $200 M_{\odot}$, quite close to the virial mass of $250 M_{\odot}$ (Mauersberger et al. 1986). If the column density estimated by Jaffe, Becklin, and Hildebrand (1984) from submillimeter data is used to derive a mass for the disk, the result is $100 M_{\odot}$ because their determination of the column density is half that of Mauersberger et al. The "dominant center" of $\mathrm{H}_{2} \mathrm{O}$ maser activity (Downes et al. 1979; Schneps et al. 1981), which exhibits by far the greatest concentration of masing spots in this complex region, is located 1 " -2 " south of this postulated, very luminous central star or dense cluster of stars.

In this picture, the compact $\mathrm{H}$ II region coincident with the northwestern part of the $12 \mu \mathrm{m}$ source is the highest electron density portion of an ionization front of a much larger $\mathrm{H}$ II region lying to the northwest of the infrared source. The compact $\mathrm{H}$ II region has a similar size, shape, and orientation to our $12 \mu \mathrm{m}$ source, but the centroids of the infrared 
emission and the radio continuum emission do not coincide; there is a $4^{\prime \prime}$ displacement between them, with the $\mathrm{H}$ II region located to the northwest. The warm $(40 \mathrm{~K})$ ammonia observed by Ho, Genzel, and Das (1983) with high resolution is also displaced from the radio continuum and lies near the $8 \mu \mathrm{m}$ peak (Genzel et al. 1982). The $12 \mu \mathrm{m}$ emission may coincide with the dense ammonia core and the $8 \mu \mathrm{m}$ peak.

Recent full synthesis mapping of the radio emission from the W51 complex by Dreher (1987) with the VLA demonstrates the existence of a morphologically complex ionized region which lies northwest of the infrared source. Ionization of this large $\mathrm{H}$ II region requires a cluster of $\mathrm{O}$ and $\mathrm{B}$ stars in a relatively low density environment. We believe that the compact H II region found by Genzel et al. (1981) marks the interface between the ultradense W51 IRS 2 core and the large-scale, extended $\mathrm{H}$ II region. The presence of the $\mathrm{H}$ II region to the northwest of the infrared source results in a lower foreground column density toward this direction; higher foreground column densities in the opposite direction, toward the southeast, may be responsible for the deep silicate absorption seen in that direction. Support for this picture is provided by the sub-millimeter maps of Jaffe, Becklin, and Hildebrand (1984) who show that the column density of cool gas and dust increases to the southeast, toward W51 IRS 1.

\section{CONCLUSIONS}

Our high-resolution $12 \mu \mathrm{m}$ image of W51 IRS 2 reveals a symmetric, dumbbell-shaped distribution in the intensity of the emitted radiation. This structure has the appearance of an edge-on disk of radius $2 \times 10^{17} \mathrm{~cm}$ and thickness $1 \times 10^{17} \mathrm{~cm}$. Its size and integrated fiux are consistent with emission from hot dust in radiative equilibrium with a central source whose luminosity is $3 \times 10^{6} L_{\odot}$. The large surface brightness of this source agrees with earlier suggestions that it is optically thick to $12 \mu \mathrm{m}$ radiation. The inferred dust temperature of $\sim 150$ $\mathrm{K}$ and high opacity are consistent with the temperature and high density $\left(n \geq 10^{7} \mathrm{~cm}^{-3}\right)$ determined from ammonia observations. A disk of density $10^{7} \mathrm{~cm}^{-3}$ and the observed extent would have a mass of order $200 M_{\odot}$, in good agreement with the virial mass (Mauersberger et al. 1986). We propose that the dominant source of luminosity is a single, very luminous source or a dense cluster of sources at the center of the disk. This object is presumably the origin of the energetic outflow revealed by the intense maser activity.

We thank Don McCarthy, Mike Cobb, and the staff of the Steward Observatory for their help with these observations. The development of the camera has been made possible by the collaboration of Steven Gaalema at Hughes Aircraft, who made available the detector used for these observations. We also wish to thank Michael Peck for his role in the camera development and Garrett Jernigan for leading the software development for this project. J. B. wishes to thank the Aspen Center for Physics for support while these observations were made. Part of this work was performed under the auspices of the US Department of Energy at the Lawrence Livermore National Laboratory under contract W-7405-Eng-48.

\section{REFERENCES}

Arens, J. F., Jernigan, J. G., Peck, M. C., Dobson, C. A., Kilk, E., Lacy, J., and Gaalema, S. 1987, Appl. Optics, in press.

Beckwith, S., and Zuckerman, B. 1982, Ap. J., 255, 536.

Bieging, J. 1975, in H II Regions and Related Topics, ed. T. L. Wilson and D. Downes (Berlin: Springer-Verlag), p. 443.

Downes, D., Genzel, R., Moran, J. M., Johnston, K. J., Matveyenko, L. I., Kogan, L. R., Kostenko, V. I., and Ronnang, B. 1979, Astr. Ap., 79, 233.

Dreher, J. 1987, private communication.

Erickson, E. F., and Tokunaga, A. T. 1980, Ap. J., 238, 596.

Genzel, R., Becklin, E. E., Wynn-Williams, C. G., Moran, J. M., Reid,

M. J., Jaffe, D. T., and Downes, D. 1982, Ap. J., 255, 527

Genzel, R., and Downes, D. 1977, Astr. Ap. Suppl., 30, 145.

Genzel, R., et al. 1981, Ap. J., 247, 1039.

Hackwell, J. A., and Gehrz, R. D. 1974, Ap. J., 194, 49.

Hackwell, J. A., Grasdalen, G. L., and Gehrz, R. D. 1982, Ap. J., 252 250.

Harvey, P. M., Joy, M., Lester, D. F., and Wilking, B. A. 1986, Ap. J., 300, 737 .

Hildebrand, R. H. 1984, Quart. J.R.A.S., 24, 267.

Ho, P. T. P., Genzel, R., and Das, A. 1983, Ap. J., 266, 596.

Jaffe, D. T., Becklin, E. E., and Hildebrand, R. H. 1984, Ap. J. (Letters), 279, L51.

Leggett, S. K. 1985, Astr. Ap., 153, 273.

Mauersberger, R., Henkel, C., and Wilson, T. L. 1987, Astr. Ap., 173, 352.

Mauersberger, R., Henkel, C., Wilson, T. L., and Walmsley, C. M. 1986, Astr. Ap., 162, 199

Schneps, M. H., Lane, A. P., Downes, D., Moran, J. M., Genzel, R., and Reid, M. J 1981, Ap, J., 249, 124

Scoville, N. Z., and Kwan, J. 1976, Ap. J., 206, 718.

Thronson, H. A., Jr., and Harper, D. A. 1979, Ap. J., 230, 133.

Walker, R. G., and Price, S. D. 1975, The AFCRL Infrared Sky Survey (Hanscom AFB, Mass.: AFGL)

JoHN F. ARENS: Space Sciences Laboratory, University of California, Berkeley, CA 94720

Roger BALL: Institute of Geophysics and Planetary Physics, L-413, Lawrence Livermore National Laboratory, PO Box 808, Livermore, CA 94550

John BaLlY: AT \& T Bell Laboratories, HOH L-245, Crawfords Corner Road, Holmdel, NJ 07733

ROBERT BeCKER: Department of Physics, University of California, Davis, CA 95616

JoHN LACY: Department of Astronomy, University of Texas, Austin, TX 78712 